\title{
Le World University Service et l'Amérique latine
}

Paola Bayle

National Scientific and Technical Research Council, Argentine

Université Nationale de Cuyo, Argentine

\section{Résumé}

Le World University Service, organisation internationale créée en 1920, œuvrait dans trois domaines distincts : l'éducation, l'aide au développement et les droits de l'homme. Dans les années 1950, son champ d'activité principal se recentra sur les pays du Sud, soit l'Asie, l'Afrique et l'Amérique Latine. Dans cet article, nous analysons les activités philanthropiques du World University Service en Amérique Latine à partir des années 1970, pour nous attacher aux caractéristiques de ses différents programmes d'assistance, de son organigramme, de l'origine des fonds recueillis et des objectifs des politiques que l'organisation a mises en place.

Mots-clés : Latino-américain - World University Service - Développement - Droits de l'homme Démocratie.

\author{
Juan José Navarro
}

Université Nationale de Cuyo, Argentine

\section{Abstract}

The Itinerary of World University Service in Latin America

The World University Service was an international organization, created in 1920, which worked in three distinctive areas: education, development and human rights. From the 1950s its main area of activity veered towards Southern countries, in Asia, Africa, and Latin America. In this paper we analyze the philanthropic work of WUS in Latin America from the seventies of the twentieth century, characterizing the variety of assistance programs, organizational structure, the source of funding and policy objectives.

Keywords: Latin American - World University Service - Development-Human Rights-Democracy. 
$\mathrm{P}$ ar tradition, les initiatives philanthropiques sont lancées en vue de contribuer à remédier à des problèmes urgents ou épineux auxquels les États échouent à répondre de façon satisfaisante. Alors que pendant la fin du XIX ${ }^{\mathrm{e}}$ siècle, qui vit l'avènement de la philanthropie moderne, un certain nombre d'organisations commençaient à s'attaquer à des questions d'ordre social résultant de l'industrialisation et des processus de développement liés à l'urbanisation, au début du $\mathrm{Xx}^{\mathrm{e}}$ siècle, les guerres ont également déclenché des actions philanthropiques.

L'une des réponses apportées dans ce domaine fut la création du World University Service (WUS), fond d'entraide universitaire à vocation mondiale, qui peut être considéré comme faisant partie d'un ensemble d'organisations non gouvernementales ayant émergé dans le secteur universitaire - certaines d'entre elles approchant les questions à traiter sous un angle religieux - afin de fournir assistance et soutien, la plupart du temps à des personnels universitaires dans le besoin. L'évolution du WUS a connu de nombreux changements et des projets, des objectifs, des modes de pensée et des champs d'action variés. Sous d'autres appellations et avec d'autres finalités, ses origines remontent aux premières décennies du Xx ${ }^{\mathrm{e}}$ siècle en Europe. Dans les années 1950, le wUS a engagé un processus d'internationalisation, déployant ses efforts en Asie, en Afrique et en Amérique latine. Cet article se concentre sur les actions de l'organisation dans cette dernière région du monde et montre la façon dont l'importance de l'Amérique latine a grandi au sein du wus dans le dernier tiers du $\mathrm{XX}^{\mathrm{e}}$ siècle. À cette fin, il s'avère nécessaire de résumer sa trajectoire (et d'y inclure les réseaux de ses contacts et ses projets phares). Nous avons examiné les opérations menées par cette organisation, en nous focalisant sur deux cas que nous considérons comme emblématiques de l'action du wUS en Amérique latine : les soutiens apportés aux universitaires chiliens à compter du coup d'État de 1973 et à la révolution sandiniste de 1979. En resserrant notre étude sur ces deux exemples, il est possible non seulement de visualiser les efforts spécifiques consentis par une ONG pour porter assistance à un groupe touché - en mettant en évidence les liens entre philanthropie, politique et idéologie -, mais également d'éclairer les changements subis par les membres des associations philanthropes sur le terrain au vu de leur implication dans ces projets. Ces agents ont été influencés par l'Amérique latine et ont vu leur carrière modifiée du fait de leur cheminement vers un état d'esprit plus militant et transnational.

\section{Le processus de création du wus}

La Première Guerre mondiale poussa un groupe d'étudiants britanniques à mettre sur pied en 1920 un fonds d'entraide aux étudiants européens - le European Student Relief (ESR) - destiné en premier lieu à venir en aide aux étudiants touchés par ce conflit armé. L'ESR a été 
créé comme l'antenne ${ }^{1}$ d'un projet antérieur, la Fédération universelle des Associations chrétiennes d'étudiants - la World Student Christian Federation (WSCF) - fondée en 1895 et basée à Genève. Cette organisation regroupait des chrétiens appartenant à un certain nombre d'Églises - catholique, protestante, pentecôtiste, orthodoxe, anglicane ou autres - et officiait au plan local comme au plan national par le biais de ce qu'on appelait les Mouvements étudiants chrétiens et qui étaient majoritairement composés d'étudiants.

Dans le milieu des années 1920, l'ESR commença à prendre ses distances avec la WSCF et, en 1926, elle changea son nom pour devenir l'International Student Service (ISS) - élargissant effectivement ses objectifs, les populations ciblées et ses champs d'action. À titre d'exemple, les programmes de l'ISS ont fourni une aide aux étudiants frappés par le tremblement de terre de 1928 en Bulgarie, les inondations de 1931 en Chine, la guerre sino-japonaise en 1937, ainsi que les luttes et les persécutions raciales et politiques en Autriche et en Allemagne sous le régime nazi.

Pendant la Seconde Guerre mondiale, l'ISS s'associa avec Pax Romana ${ }^{2}$ et à nouveau avec le

1 Pour cette raison, de nombreux membres du wus ont rejoint la structure organisationnelle de l'ESR. En effet, Ruth Rouse, membre du WSCF a joué un rôle actif de premier ordre au moment du lancement de l'ESR et Conrad Hoffman, secrétaire général du WSCF, fut nommé premier dirigeant de l'ESR.

2 Pax Romana est une autre de ces organisations catholiques étudiantes qui s'est distinguée au cours de la
WSCF pour mettre en place le Fonds d'entraide aux étudiants européens - le European Student Relief Fund (ESRF) - en vue de reconstruire les universités frappées durant la guerre - au détriment d'autres projets tels que la création d'une université internationale - et d'aider des centaines d'anciens prisonniers à terminer leurs études supérieures. En 1943, l'ESRF changea de nom pour devenir le WSR - le World Student Relief. Au cours de ces nouvelles missions, l'ISS assura une fonction de coordination en gérant des tâches administratives.

Plusieurs organisations engagées dans des initiatives communes ont conservé leur propre voie autonome, à la différence de L'Iss et du WSR, qui, en 1950, fusionnèrent en une organisation non gouvernementale appelée World University Service/Entraide universitaire mondiale (EUM).

\section{Organigramme du wus}

Au sein du wUS, des Comités nationaux - d'abord mis en place par des agents des universités ou d'autres organisations dans leur pays respectif ont servi d'unités de fonctionnement de base en assurant l'exécution des programmes d'action. Il existait également trois autres « formes d'engagement : des comités de correspondants, des groupes de contact et des membres associés $»^{3}$. Les Comités nationaux se retrouvaient

période de l'entre-deux-guerres. S'inscrivant dans la continuité de son prédécesseur Auxilium Studiorum, Pax Romana offrait une aide aux étudiants touchés par la Première Guerre mondiale.

3 International wus, What is wus? (Geneve: wus, 1992), p. 2. 
pendant l'Assemblée générale, instance supérieure qui se réunissait tous les trois ans afin de tracer les grandes lignes des politiques globales. Les membres qui siégeaient dans les Comités nationaux étaient les seuls à posséder un droit de vote. À son tour, cette instance supérieure élisait un Comité exécutif composé de treize membres qui représentaient de façon équitable les quatre régions du monde (Afrique, Amérique latine et Caraïbes, Europe et Amérique du Nord, enfin Asie et Pacifique) afin qu'il mette en place les politiques établies par l'Assemblée. Le Secrétariat international basé à Genève jouait un rôle clé dans la structure organisationnelle du wus puisqu'il avait à sa tête un Secrétaire général qui coordonnait la création des nouveaux Comités nationaux, promouvait la création de réseaux en lien avec d'autres organisations et représentait le wus lors de forums internationaux. Enfin, un bureau exécutif, constitué d'un président, d'un vice-président, d'un trésorier et d'un secrétaire général formait un degré intermédiaire entre le Comité exécutif du wUS et le Secrétariat international.

Chaque Comité national se trouvait à la tête de programmes du wus développés sur la base de sa localisation et de ses besoins spécifiques, accordant, dans certains cas, une assistance à des réfugiés, alors que dans d'autres il soutenait des universités. Autrement dit, les Comités nationaux agissaient comme des entités relativement autonomes qui suivaient les grandes orientations définies par l'Assemblée générale.
Tout comme il avait revu son appellation, le wUS révisa certains de ses objectifs et de ses perspectives. Une nouvelle période d'initiatives transnationales s'ouvrit pour l'organisation qui se concentra sur l'Afrique, l'Asie et, plus tard, l'Amérique latine - faisant ainsi porter ses efforts sur les problèmes du Tiers-monde. Ainsi, le processus d'internationalisation du wuS prenait de l'ampleur puisque le champ d'action de ses initiatives n'était plus restreint aux nations européennes. Dans le contexte de la Guerre froide, marqué par les processus de décolonisation, les luttes pour l'indépendance et la création de multiples agences internationales ou multilatérales destinées à édifier une base de travail en vue d'une compréhension et d'une coopération mutuelles, une nouvelle approche fut définie pour analyser la responsabilité sociale des universités. Le wUS, du moins dans son discours, garda ses distances par rapport aux querelles idéologiques. Étroitement liée à une tradition œcuménique, l'organisation défendait la perspective d'une communion internationale. Partant, elle déploya des programmes d'entraide en faveur des étudiants algériens réfugiés en Tunisie et au Maroc (1958) et des étudiants tchécoslovaques accueillis au RoyaumeUni (1968), entre autres. Malgré sa prise de position en faveur de la neutralité politique, elle afficha à partir des années 1970 une position idéologique de plus en plus radicale.

Au cours de son Assemblée générale à Grenoble, en 1952, l'organisation édifia son premier Programme d'action en déclarant : 
"L'idée-force du concept du wUS se trouve résumée dans l'expression : solidarité estudiantine internationale par le biais du service mutuel. La relation mutuelle au service commun remplace la relation du donateur au récipiendaire et confère déjà une vitalité nouvelle aux travaux du wUS, car chacun a quelque chose à donner, chacun a quelque chose à recevoir $»^{4}$.

Dans le même temps, le wus établit un certain nombre de critères propres à l'approbation d'un projet, critères articulés, dans les années 1950, autour de quatre thèmes : l'hébergement et la vie courante ; la santé des étudiants ; les actions éducatives et les infrastructures; le secours aux personnes et l'aide d'urgence. Ces préoccupations étaient principalement liées au personnel universitaire.

Ces grandes lignes furent bousculées par des contextes locaux disparates. En Afrique, où les programmes ont d'abord été introduits en 1952, les objectifs clés du WUS consistaient à rapprocher les universités des communautés et à fournir une aide aux réfugiés étudiants ainsi qu'aux victimes de l'apartheid, sur fond de discrimination raciale et de ségrégation. De 1981 à 1992, cet axe de travail prit un nouvel élan avec la création, par le wUS, du Programme de bourses pour l'Afrique du Sud et la Namibie - le South Africa and Namibian (SAN) Scholarship Programme ${ }^{5}$.

À son tour, le paysage universitaire asiatique présenta un

4 International wus, 50 years (Genève: wus, 1970), p. 32.

5 International wus, What is wus?, op. cit. (cf. note 3).
" surplus d'institutions dédiées à l'enseignement supérieur, ce qui engendr[a] des négligences déplorables quant aux installations destinées aux étudiants et affect[a] la qualité de l'enseignement universitaire $»^{6}$.

Jusqu'en 1970, les programmes lancés par le wUS en Asie consistaient en majeure partie en une aide matérielle affectée aux infrastructures universitaires ou appelée à aider les étudiants.

En dernier lieu, l'Amérique latine figura également au programme des dispositifs du wUS en 1960. Cet ajout tardif - au regard d'autres régions du monde - était dû au fait que le wUS entretenait peu de liens avec des agents locaux capables de s'investir dans le développement d'un programme. L'événement qui consolida l'implantation du wus dans cette région fut le tremblement de terre de Valdivia (Chili), qui frappa l'Université de la Conception et l'Université australe. En outre, le wUS finança un projet de développement de la communauté, lancé par le Département pour l'aide sociale de l'Université du Chili en $1965^{7}$. Ce projet visait à intégrer l'université à d'autres secteurs de la société, par des collaborations avec des groupes sociaux - ouvriers ou agriculteurs par exemple au moyen d'activités destinées à les informer ${ }^{8}$.

Le tableau ci-dessous résume l'intégralité des principaux programmes lancés par le wus dans

6 International wus, 50 years, op. cit., p. 32 (cf. note 4).

7 University Decree $n^{\circ}$ 1562, en date du 14 avril 1965.

8 Project $n^{r} 3.30$, Chile, Community Development in collaboration with Universidad de Chile's Department of Social Aid, wus, 1971. 
ces régions jusqu'en 1970 afin de montrer le poids initial limité de l'Amérique latine, région que nous examinons plus en détail, dans le cadre de la politique du wus. Cet état de fait commença à évoluer au cours des années 1970, époque à laquelle l'Amérique latine revêtit une plus grande importance pour le wUS.

\section{Les grands thèmes abordés par le wus}

Depuis le début, le wus s'est focalisé sur l'aide apportée aux réfugiés étudiants - à comprendre dans une acception large et non dans un sens strictement légal -, activité poursuivie jusqu'à une période tardive du $\mathrm{Xx}^{\mathrm{e}}$ siècle. En outre, le wUS a également introduit d'autres objectifs comme la promotion du développement, ou la réaffirmation et le renforcement des droits de l'homme et de la démocratie. Ces objectifs furent intégrés à la plupart de ses initiatives.

Comme d'autres organisations qui firent $d u$ développement le thème moteur de leur profession de foi, le wUS inscrivit cette question à son ordre du jour. Dans la formulation d'une idéologie du développement, des jalons avaient été posés : le discours sur l'état de l'Union du président américain Harry Truman en 1949, qui vit l'amorce de ce que l'on appelait l'Âge du développement ${ }^{9}$, la déclaration de l'Assemblée générale des Nations unies (ONU) en 1961, qui lança la Première décennie pour le développe-

9 Gilbert Rist, El desarrollo: historia de una creencia occidental, Madrid, Los Libros de la Catarata, 2002. ment de l'oNU ${ }^{10}$, la présentation, en 1968, du rapport de la Commission Pearson sous l'intitulé Partenaires pour le développement ${ }^{11}$, ainsi que la résolution 2626 (XXv) de l'oNU, adoptée en 1970, qui proclamait le début de la Seconde décennie pour le développement ${ }^{12}$. Ces déclarations et résolutions visaient à élaborer un schéma général pour le développement correspondant aux critères des principales grandes puissances. En effet, le « développement » faisait partie de l'agenda des deux blocs au cours de la Guerre froide, chaque superpuissance soutenant une définition différente du terme et des critères divergents pour l'atteindre ${ }^{13}$. Le wUS rejoignit un nombre d'organisations internationales occidentales - et pas seulement américaines - qui soutenaient des programmes centrés sur cette question. Les fonds reçus par le wus de la part de plusieurs agences gouvernementales pour le développement l'obligèrent en grande partie à engager des initiatives plus avant dans cette voie.

10 ONU. Résolution 1710 (XVI) UN Decade for Development, 1961.

11 Lester B. Pearson, ancien Premier Ministre canadien, présida cette commission qui comptait sept autres membres. Seuls deux des huit membres de la Commission venaient de pays en voie de développement : le Brésilien Roberto de Oliveira Campo et Arthur Lewis, originaire de St Lucie. Les autres membres de la Commission représentaient les États-Unis, le Royaume-Uni, la RFA, la France, le Japon et le Canada.

12 ONU. Résolution 2626 (xxV); International Development Strategy for the United Nations' Second Decade for Development, 1970.

13 Judith Hart, Aid and Liberation. A Socialist Study of Aid Politics (London: Victor Gollancz Ltd, 1973). 
Tableau 1. Programmes internationaux du wUS

\begin{tabular}{|c|c|c|c|}
\hline Régions - Années & $\begin{array}{c}\text { Pays dans lesquels ils ont été } \\
\text { développés }\end{array}$ & Programmes mis en oeuvre & $\begin{array}{c}\text { Sommes investies } \\
\text { (dépenses exprimées en } \\
\text { francs suisses) }\end{array}$ \\
\hline $\begin{array}{c}\text { Asie } \\
\text { 1955-1968 }\end{array}$ & $\begin{array}{l}\text { Inde, Indonésie, Japon, } \\
\text { Pakistan, Vietnam, } \\
\text { Philippines, Ceylan, Corée } \\
\text { du Sud, Hong-Kong, Népal }\end{array}$ & $\begin{array}{l}\text { Santé des étudiants, centres et } \\
\text { coopératives pour les } \\
\text { étudiants, foyers, cantines, } \\
\text { magasins, infrastructures } \\
\text { éducatives, subventions et } \\
\text { frais de service }\end{array}$ & 4993915 \\
\hline $\begin{array}{c}\text { Afrique } \\
1952-1968\end{array}$ & $\begin{array}{c}\text { Algérie, Rhodésie, Afrique } \\
\text { du Sud, Burundi, Lesotho, } \\
\text { Ouganda, Botswana, } \\
\text { Swaziland, Rwanda, } \\
\text { Éthiopie, Ghana, Kenya, } \\
\text { Nigéria, Malawi, Sierra } \\
\text { Leone, Tanzanie, Soudan, } \\
\text { Zambie }\end{array}$ & $\begin{array}{c}\text { Assistance auprès des } \\
\text { étudiants réfugiés, } \\
\text { bibliothèques et matériel } \\
\text { éducatif, foyers et } \\
\text { coopératives, programmes de } \\
\text { santé, fournitures médicales }\end{array}$ & 4464720 \\
\hline $\begin{array}{c}\text { Amérique latine } \\
\text { 1961-1969 }\end{array}$ & $\begin{array}{c}\text { Pérou, Guatemala, } \\
\text { Nicaragua, Paraguay, Chili, } \\
\text { Honduras }\end{array}$ & $\begin{array}{c}\text { Centres et foyers pour } \\
\text { étudiants, centres sanitaires et } \\
\text { cliniques, infrastructures et } \\
\text { équipements éducatifs, } \\
\text { bourses et subventions }\end{array}$ & 901495 \\
\hline
\end{tabular}

Source : Tableau élaboré par les auteurs à partir d'éléments recueillis auprès du WUS, Action, vol. xx, World University Service, 1970/1. 
Au reste, la défense des droits de l'homme dans des domaines différents (éducation, vie de la communauté, etc.) fit également figure de pierre angulaire dans les actions du wUS au cours de la seconde moitié du Xx ${ }^{\mathrm{e}}$ siècle. À cette fin, l'organisation contracta un certain nombre d'alliances avec des activistes, des agences intergouvernementales et d'autres ONG, bâtissant selon les termes de Keck et Sikkink ${ }^{14}$, des réseaux de défense des droits. L'implication du wus en faveur des droits de l'homme s'étendit pour englober d'autres questions qui furent traitées de concert - comme l'éducation et la démocratie. Ainsi, le wus s'attacha, d'une part, à promouvoir l'accès à l'éducation comme un des droits de l'homme. Alors que, durant les premières années, le wUS avait fait de l'enseignement supérieur l'une de ses principales priorités, il adopta ensuite un point de vue holistique sur l'éducation pour tous lorsqu'il souscrivit à la Déclaration de New Delhi en 1991. D'autre part, il encouragea la démocratisation et la promotion des droits de l'homme dans les universités, s'inspirant d'un point de vue conceptuel et idéologique de la Déclaration de Lima de 1988 sur l'autonomie et la liberté pédagogiques des institutions de l'enseignement supérieur ${ }^{15}$.

14 Margaret Keck, Kathryn Sikkink, Activist Beyond Borders: Advocacy Networks in International Politics (New York: Cornell University Press, 1998).

15 La Déclaration de Lima fut adoptée par le wus lors de son Assemblée générale en 1988. Ce document appelle à l'autonomie de l'enseignement universitaire, à la noninterférence en matière d'éducation et d'enseignement, à la liberté de conserver des liens internationaux, aux droits des étudiants, et à la liberté d'association pour toutes les
En Amérique latine, l'engagement du wuS pour les processus de retour à la démocratie suite à l'interruption du fonctionnement des institutions - coups d'État ou guerres civiles - était sous-tendu par des initiatives visant à rétablir la démocratie et camouflées sous forme d'actions humanitaires. Le soutien de l'organisation pour cette cause englobait un certain nombre de projets. Ses programmes d'assistance et de retour en faveur des réfugiés apportaient un soutien à une opposition politique hostile à la dictature. Comme l'ont noté Peck et Vilas, « apporter un soutien aux représentants de l'opposition (démocratique) et leur permettre de servir leur engagement politique à l'étranger constituent, presque en soi, un soutien envers la démocratie ${ }^{16}$. De même, les programmes internes du wUS visaient à renforcer la démocratie au sein de la vie sociale et jouaient un rôle moteur quant aux actions de soutien en faveur de l'égalité entre les sexes, de l'éducation et des populations locales, entre autres.

Comme nous allons le montrer, ces préoccupations, à l'œuvre dans les projets menés en Asie et en Afrique, se sont également traduites dans le travail engagé par le wUS en Amérique latine.

institutions d'enseignement supérieur. Lors de la même Assemblée, le wus créa son Programme global des droits de l'homme pour l'éducation. Le wus considérait la liberté d'enseignement comme un droit de l'homme et l'éducation comme un droit non exclusivement réservé à une élite mais qui devait profiter à l'ensemble de la société (International wUs, What is wUS?, op. cit., cf. note 3).

16 Lennart Peck, Carlos Vilas, Servicio Universitario Mundial (WUS) en América Latina: programa de becas para refugiados, Estocolmo, SIDA, 1997, p. 19. 


\section{Du Chili au Nicaragua}

L'avènement du wUS en 1950 fut marqué par l'élargissement et l'internationalisation de ses actions en dehors de l'Europe. Celles-ci se concentrèrent d'abord sur les pays en voie de décolonisation du sud de l'Afrique ou en Asie. L'organisation s'implanta ensuite en Amérique latine en 1960, et pendant un peu plus d'une dizaine d'années déploya ses programmes dans une poignée de nations latino-américaines. Compte tenu de son champ d'action plus restreint, le réseau de contacts du wUS avec les agents sur place s'avéra limité en comparaison des liens créés dans d'autres régions. Jusqu'au début des années 1970, les seuls développements dignes d'attention furent la création du Comité national du wUS du Nicaragua et un programme d'entraide de petite envergure en faveur des réfugiés brésiliens au Chili en $1972^{17}$.

C'est à la suite du coup d'État militaire au Chili le 11 septembre 1973 que le wus devint un acteur de poids en Amérique latine. Le bombardement du Palais de la Moneda à Santiago - où siégeait le gouvernement - et la fin de l'expérience sans précédent d'un gouvernement socialiste démocratique alimentèrent de multiples

17 Ce programme venait en aide aux ressortissants brésiliens affectés par le coup d'État militaire qui renversa le président Joao Goulart en 1964. Celui-ci serait « le premier d'une série de coups d'État montés en Amérique latine par des militaires en tant qu'institution vouée à changer radicalement non seulement les politiques économiques et sociales mais également le système politique au niveau local » (Leslie Bethell (dir.), Historia de América Latina, Barcelona, Editorial Crítica, 1990, vol. 12, p. 49). efforts de solidarité destinés à apporter une aide. La « Voie chilienne vers le socialisme » de Salvador Allende avait éveillé empathie et soutien au sein des mouvements de gauche, dans les rangs des progressistes et des syndicats. Les visées de l'Unité populaire - coalition politique dirigée par Allende - et le violent coup d'État avaient conféré au Chili une visibilité sans précédent. Contester le régime instauré par Augusto Pinochet Ugarte (1973-1990) devint une

« cause célèbre pour les Européens et rencontra un écho important auprès des fonctionnaires, des représentants parlementaires, des militants des partis politiques, des dirigeants syndicaux des groupes de défense des droits de l'homme, des Églises protestantes et catholiques et des fédérations d'étudiants $»^{18}$.

Alan Angell ${ }^{19}$ a procédé à une analyse des fonds reçus par les opposants au régime de Pinochet lorsque ce dernier était en place, argent principalement destiné à des fins politiques mais également voué à des fins humanitaires, éducatives ou autres. Ces fonds provenaient de plusieurs sources, mais ils étaient en majeure partie accordés par des organismes publics, des partis

18 Mario Sznajder, Luis Roniger, "Exiles Communities and their Differential Institutional Dynamics: A Comparative Analysis of the Chileans and the Uruguayan Political Diaspora”, Revista de Ciencia Política, vol. 27 (2007/1) p. 50.

19 Alan Angell, "International Support for the Chilean Opposition, 1973-1989: Political Parties and the Role of Exile", in The International Dimensions of Democratization: Europa and the Americas, Oxford Studies in Democratization (Oxford: Oxford University Press, 1996). 
politiques et des syndicats, et transitaient par des ONG opérant à la fois au Chili et ailleurs. Les pays d'Europe du Nord, les Pays-Bas, les partis politiques et les syndicats italiens ainsi que les fondations allemandes à visée philanthropique se démarquent parmi les contributeurs eu égard au montant de leur participation financière. L'internationalisation d'une cause nationale - le retour de la démocratie au Chili était aussi le fait d'émigrés chiliens ${ }^{20}$ qui mirent en place des "cellules de l'esprit chilien ${ }^{21}$ dans leur pays d'accueil, faisant de leur exil une puissante force de combat contre le régime de Pinochet. Comme Sikkink le notait, une communauté politique fermée - en l'espèce la dictature chilienne - favorise les liens transnationaux, puisqu'elle précipite les opposants locaux

«à l'étranger - parfois même pour protéger sa propre existence. L'un des enjeux majeurs pour les actions internationales est de plaider en

20 Les estimations de l'émigration chilienne après le coup d'État de 1973 sont très variables : le Bureau national de retour au Chili les chiffre à environ 200000 personnes en 1990, le Vicariat de la solidarité au Chili à environ 260000 (Loreta Rebolledo, Memorias del Desarraigo, Testimonios de exilios y retorno de hombres y mujeres de Chile, Santiago de Chile, Catalonia, 2006). Certains auteurs avancent le chiffre de 408000 (Carmen Norambuena, "Exilio y retorno. Chile 1973-1994 ", in Mario Garcés, Pedro Milos, Myriam Olguín, Julio Pinto, María Teresa Rojas, Miguel Urrutia [dir.], Memoria para un nuevo siglo. Chile, miradas a la segunda mitad del siglo XIX, Santiago de Chile, LOM Ediciones, 2000), voire d'un million (Fernando Montupil, Exilio, Derechos Humanos y Democracia, El Exilio Chileno en Europa, Santiago de Chile, Coordinación Europea de Comites Pro Retorno, 1993).

21 Jorge Arrate, Exilio. Textos de denuncia y esperanzas, Santiago de Chile, Ediciones Documentas, 1987. faveur de la libéralisation et de l'ouverture des régimes nationaux $»^{22}$.

En conséquence, une cause nationale - la lutte contre la dictature chilienne - a franchi les frontières nationales, sous l'impulsion des liens établis entre les Chiliens (émigrés ou citoyens qui sont restés au pays) et toutes les personnes favorables à la résistance contre Pinochet.

Dans ce contexte, le wUS élabora son premier programme d'importance en Amérique latine : le Chilean Refugee Scholarship programme (CRSP), lancé par la branche anglaise du wUS (WUS UK). La création de ce programme était motivée par le souci des universitaires britanniques qui se regroupèrent au sein de l'Academics for Chile (AFC), mouvement universitaire pour le Chili, placé sous la direction d'Alan Angell, de St. Anthony College, de l'Université d'Oxford (secrétaire exécutif), de Christian Anglade, du Département de science politique de l'Université d'Essex (président) et de David Rock, directeur du centre des Études latino-américaines de l'Université de Cambridge (trésorier). Ce groupe parvint à mobiliser des intellectuels qui avaient précédemment entretenu des liens avec le Chili et/ou l'Amérique latine, comme Ann Zammit et Emanuel de Kadt, ralliant également d'autres universitaires ${ }^{23}$ qui condamnaient les attaques

22 Kathryn Sikkink, « La dimensión transnacional de los movimientos sociales », in Elizabeth Jelin (dir.), Más allá de la nación : las escalas múltiples de los movimientos sociales, Buenos Aires, Libros del Zorzal, 2003, p. 301-333.

23 Comme toutes les organisations britanniques, I'AFC avait des sponsors : Alec Nove, professeur d'économie inter- 
contre le milieu universitaire chilien et les violations des droits de l'homme. Après le coup d'État, ces universitaires ont commencé à se mobiliser et à promouvoir des actions de solidarité qui se sont d'abord articulées autour d'appels à l'aide en faveur des universités, afin que ces institutions aident les étudiants chiliens résidant au Royaume-Uni à y demeurer si la bourse qui leur avait été octroyée était menacée par l'évolution de la situation politique.

Le cas du Chili dépassait le cadre d'action de ce mouvement informel. En conséquence, l'AFC se tourna vers le wUS UK au regard de la plus grande expérience de ce dernier en matière d'aide aux universitaires réfugiés ${ }^{24}$. Lorsque le parti travailliste remporta l'élection géné-

nationale à l'Université de Glasgow ; Kenneth Kirkwood, professeur de "Race relations" à l'Université d'Oxford ; Martin Pollock, professeur de biologie moléculaire à l'Université d'Édimbourg ; Henri Tajfel, professeur de psychologie sociale à l'Université de Bristol ; Dudley Seers, chercheur à l'Institut d'Études du développement ; Raymond Williams, de Université de Cambridge ; Richard Kahn, professeur d'économie à l'Université de Cambridge ; Norman Dombey, de l'École de mathématiques et de physique à I'Université du Sussex; Peter Flynn au Centre pour les études latino-américaines, à I'Université de Glasgow ; Walter Little, du Département de science politique de I'Université de Liverpool ; Charles Posner, de l'Institut de l'éducation à l'Université de Londres ; lan Wright, du Queen's College, de l'Université de Cambridge et David Winder, du Département des études de management de I'Université de Manchester.

24 En 1973, le wUS UK développait des programmes pour les réfugiés ougandais au Royaume-Uni et pour les étudiants tchécoslovaques après le Printemps de Prague. On trouva une place à de nombreux étudiants dans des lycées, des écoles polytechniques et les universités quel que soit le niveau de leur cursus. rale de 1974, le wUS UK obtint des fonds du ministère du Développement de l'outre-mer (Overseas Development Ministry-ODM) afin de bâtir un programme plus conséquent. En effet, jusqu'en février 1974, le gouvernement britannique, dirigé par les conservateurs à l'époque, n'autorisait pas l'accueil des réfugiés chiliens sur son sol et maintenait, en fait, des liens amicaux avec le régime militaire chilien. Le tournant politique au Royaume-Uni changea la donne pour les Chiliens exilés. Les actions de solidarité menées en faveur du peuple chilien par les syndicats, les défenseurs des droits de l'homme et les partis politiques, voyaient ainsi s'ouvrir un espace politique leur donnant une légitimité. Plusieurs membres du parti travailliste britannique - alors au gouvernement - avaient conservé des liens politiques avec l'administration déchue de Salvador Allende et avaient sympathisé avec sa « Via chilena al socialismo » ( "Voie chilienne vers le socialisme »). Judith Hart, ministre du Développement de l'outremer, fut un membre essentiel de ce groupe. De 1970 à 1973, Hart prit publiquement la défense du projet d'Allende et, après le coup d'État, devint une opposante farouche et éloquente à la dictature chilienne. Suite à sa nomination en qualité de ministre, des membres du WUS UK et de l'AFC, au rang desquels Dudley Seers, ancien directeur général de la planification économique du ODM, avaient la certitude qu'un nouveau soutien financier serait alloué 
à la cause chilienne ${ }^{25}$. Hart réaffecta les fonds initialement attribués au Chili pour une aide au développement et les consacra au programme du WUS UK, permettant à environ 900 Chiliens de poursuivre des études au Royaume-Uni 473 suivaient un cursus de second et troisième cycles universitaires, 343 visaient un diplôme de premier cycle et 84 étaient inscrits dans l'enseignement technique. Parmi tous les bénéficiaires, $64 \%$ avaient terminé leurs cursus en 1986.

De 1974 à 1986, le CRSP reçut des fonds d'un montant de $11188736 £$ de la part du gouvernement britannique pour l'aide au développement. Ainsi, le programme englobait les actions essentielles du wUS : d'une part, le programme fournissait une aide humanitaire et une assistance éducative aux réfugiés et, d'autre part, le wUS UK s'attaquait à une violation manifeste des droits de l'homme ${ }^{26}$; enfin, conformément aux exigences de son bailleur de fonds (l'oDM britannique), le

25 L'engagement de Dudley Seers au sein du Comité pour l'octroi des bourses d'études du wus se révéla déterminant lors des réunions aboutissant aux décisions de subventions, en raison de son prestige politique et universitaire.

26 Durant les 17 années que dura le régime militaire, les violations des droits de l'homme sont devenues courantes : torture, emprisonnement au mépris de l'application des lois, passages en cour martiale, enlèvements, exils intérieur ou extérieur, persécutions et meurtres ont placé le Chili - et d'autres pays comme l'Argentine, le Brésil et I'Uruguay - parmi les dictatures les plus violentes d'Amérique du Sud (Rapport publié par la Commission nationale pour la vérité et la réconciliation et connu sous le nom de Rapport Rettig, 1990 ; rapport de la Commission nationale sur les prisons politiques et la torture, 2004). programme soutenait l'idée selon laquelle le développement du Chili serait le résultat de la formation reçue au Royaume-Uni par les bénéficiaires des bourses ${ }^{27}$. Puisque cet objectif ne pouvait être rempli qu'une fois les émigrés revenus au Chili, le wUS UK élabora un programme d'aide au retour engagé une fois les conditions politiques de sa mise en place réunies. Afin d'évaluer le retour potentiel au Chili ou la relocalisation dans d'autres pays latino-américains, Marilyn Thompson (chargée du traitement du dossier au WUS UK) se rendit dans plusieurs pays de la région où elle eut des entretiens avec des universitaires, des dirigeants politiques et des défenseurs des droits de l'homme. Ainsi, elle se rendit au Panama (au Comité Pro Refugiados y Comité Ecuménico, ainsi que dans d'autres centres universitaires), au Costa Rica (à la Confederación de Universidades Centroamericanas), au Mexique (au Consejo Nacional de Ciencia y tecnología), au Vénézuela (au Centro de Estudios para el Desarrollo), en Colombie (au Centro de Estudios de Desarrollo Económico et Universidad de Los Andes), en Équateur (à la Facultad Latinoamericana de Ciencias Sociales, sede Ecuador, et au Centro de Estudios Sociales y Planificación), au Chili (à la Facultad Latinoamericana de Ciencias Sociales, sede Chile, à l'Academia de Humanismo Cristiano et à la Sociedad

27 Paola Bayle, "1973: Chilean Academics in the Emergency", Österreichische Zeitschrift für Geschichtswissenschaften, vol. 3 (2010), p. 119-145. 
de Profesionales y Técnicos), en Argentine (au CLACSO) et en Uruguay. En vertu de ce programme, 253 anciens titulaires d'une bourse du wUS UK furent relocalisés. La plupart souhaitaient retourner au Chili. 198 y parvinrent alors que 44 s'installèrent dans d'autres pays latino-américains et que 11 d'entre eux s'établirent hors d'Amérique latine ${ }^{28}$.

Géré par le Comité national du wUS UK, le programme du CRSP a donné une impulsion décisive à l'expansion de l'organisation en Amérique latine, en développant son réseau auprès d'universitaires, de militants des droits de l'homme ou encore d'organisations religieuses. Plusieurs membres du WUS UK, dont Wendy Tyndale, Pauline Martin, ou Marilyn Thompson, se rendirent en mission spéciale en Amérique latine pour évaluer la situation et trouver des soutiens au niveau local et parvinrent à créer un réseau qui comptait notamment des ambassades, le Comité intergouvernemental pour les migrations européennes (CIME), la Croix Rouge et des organismes de défense des droits de l'homme.

À partir des années 1970, la situation d'urgence vécue au Chili se propagea dans d'autres pays d'Amérique latine à la suite d'autres coups d'État militaires - en Uruguay (1973) et en Argentine (1976) - et de guerres civiles - au Salvador (1980). D’autres pays comme le Paraguay et le

28 Paola Bayle, "Back Home: the World University Service UK Return Programme For Chilean Academic Exiles", in Fernanda Beigel, ed., The Politics Of Academic Autonomy in Latin America (London: Ashgate, 2013).
Guatemala se débattaient contre une instabilité institutionnelle (des dictatures hybrides, selon les termes d'Ansaldi et de Giordano ${ }^{29}$ ), ceci alors que la violence politique sévissait dans la plus grande partie des pays latino-américains, violence soutenue financièrement par les ÉtatsUnis dans sa quête pour la préservation de son hégémonie. Dans ce contexte, la nécessité de l'aide aux réfugiés grandit et l'Amérique latine devint une priorité du wUS.

Cette évolution se confirma lors du premier Congrès des sections latino-américaines du wUS à Quito (Équateur) en 1976, au cours duquel les initiatives éparses firent place à une rationalisation des actions à l'échelle régionale. Dix Comités nationaux furent créés et le wus inaugura son Bureau régional pour l'Amérique latine grâce notamment au travail dynamique de Pauline Martin, spécialiste du continent au wUS UK. Géré par un coordinateur régional, ce bureau servait de liaison entre Genève et les multiples Comités nationaux du wUS pour l'approbation des projets soumis, leur gestion et leur exécution $^{30}$. La présence grandissante de l'Amérique latine dans le système de fonctionnement du wUS rejaillit sur le groupe conséquent qui rejoignit la session de l'Assemblée générale au Sri Lanka en 1979 - et qui représentait l'Amérique latine pour la première fois - et sur la décision

29 Waldo Ansaldi, Verónica Giordano, América Latina. La construcción del orden. Tomo II, Buenos Aires, Ariel, 2012.

30 wus International, wUS News Latin America (Switzerland: WUS, 1979). 
du wus de tenir sa première Assemblée générale en Amérique latine en 1980.

À mesure de l'expansion du wus dans la région, les Latino-Américains eux-mêmes ont commencé à travailler pour l'organisation et à diriger des programmes initialement lancés par des Européens. La structure du wUS se complexifia avec la création d'un bureau subrégional de coordination pour l'Amérique centrale, les Caraïbes et le Mexique basé à San José (Costa Rica) ; d'un bureau subrégional pour l'Amérique du Sud à Quito (Équateur) ; et enfin d'un bureau de coordination régionale pour toute l'Amérique latine à Santiago du Chili. Des universitaires latino-américains, des chefs de file de la gauche et des militants pour les droits de l'homme (tels Ricardo Lagos, ancien président chilien entre 2000 et 2006 et Jorge Taiana, ancien secrétaire exécutif de la Commission interaméricaine pour les droits de l'homme de l'Organisation des États américains, et ancien ministre argentin des Affaires étrangères entre 2005 et 2010 ) rejoignirent le wUS pour y occuper des postes de haut niveau dans leur Comité national respectif. De façon similaire, certains Latino-Américains furent nommés à des postes haut placés dans la structure organisationnelle générale du wUS, comme Félix Ulloa, originaire du Salvador, qui occupa le siège de président du bureau exécutif du wUS en 1980 et le Chilien Ximena Erazo, qui fut nommé secrétaire général adjoint.
Alors qu'elle n'avait pas été formellement reconnue lors de sa création, la dimension politique de l'action du wUS s'affirma ouvertement au milieu des années 1970, quand l'Amérique latine commença à peser de façon conséquente sur sa structure, ses efforts d'expansion, le profil politique des dirigeants et des membres des Comités nationaux et, par conséquent, sur le choix de ses programmes et leur lancement. Ainsi, les programmes de bourses ou d'assistance proposés aux réfugiés latino-américains victimes de violations des droits de l'homme ou de violences politiques allaient croissant tant pour leur nombre que pour les montants engagés. Ces initiatives étaient complétées par des programmes d'aide au retour qui n'échappaient pas aux luttes politiques de chacun des pays. Les critères de sélection des bénéficiaires ne s'articulaient pas seulement autour de conditions liées aux circonstances d'un point de vue humanitaire ou universitaire, attendu que l'affiliation politique jouait aussi un rôle clé dans la promotion des processus locaux de retour à la démocratie. Les politiques de sélection, financées par des sources liées aux partis sociauxdémocrates européens, ont aussi révélé l'interaction entre les domaines nationaux et internationaux de la philanthropie et de la politique. Comme l'a indiqué un membre latino-américain du wus dans un entretien pour cette étude, « En Amérique latine comme en Afrique, ces programmes étaient utilisés par les partis sociauxdémocrates européens pour soutenir la gauche 
latino-américaine... et c'est comme cela que ça se passait $»^{31}$.

Après la victoire de l'Armée de libération nationale (ELN) sandiniste en 1979, le Nicaragua devint, avec le Chili, un autre destinataire capital à la fois de l'aide philanthropique et du soutien politique. L'expérience unique de l'ELN marqua un changement dans le déroulement des processus politiques en Amérique latine. Ainsi, au début des années 1980, l'Amérique latine voyait se dérouler deux scénarii distincts : d'une part, le Cône Sud était tombé aux mains de régimes autoritaires, alors que, d'autre part, des luttes pour la libération, des utopies envisageables et la perspective d'une société plus égalitaire coexistaient au Nicaragua. L'ELN « se fixa quatre objectifs majeurs : démocratie, développement et transformation, bien-être social, souveraineté et auto-détermination $»^{32}$. Sur la base de ces finalités principales, l'administration sandiniste fit appel aux membres du WUS - et, de fait, aux secteurs les plus progressistes d'Europe et d'Amérique latine. C'est la raison pour laquelle un certain nombre de programmes ont été mis sur pied pour contribuer à ce processus révolutionnaire. Des documents officiels évoquaient ouvertement cette empathie : sous le titre Nicaragua. Une brèche dans l'impérialisme

31 Nous avons choisi de conserver l'anonymat de cette source.

32 Orlando Nuñez Soto, Transición y lucha de clases en Nicaragua (1979-1986), México, Siglo XXI Editores, 1987 , p. 338.
$U S$, le wUS faisait savoir que ce pays d'Amérique centrale

« représent[ait] une fissure béante dans la muraille érigée par la mainmise militaire sur la région. Pour les autres nations en lutte pour leur liberté, preuve [était] encore faite qu'il n'exist[ait] aucune dictature militaire ni aucun impérialisme capable de résister à la volonté d'un peuple uni $»^{33}$.

La convergence politique entre le wus et la révolution sandiniste donna lieu à des efforts conjoints. À la fin de l'année 1979, le gouvernement révolutionnaire du Nicaragua sollicita l'aide de l'Organisation internationale pour les migrations (OIM) en vue d'élaborer des programmes de relocalisation en faveur de Nicaraguayens exilés, d'autres LatinoAméricains qualifiés d'Européens. Parmi les priorités du gouvernement nicaraguayen, on comptait la planification et le développement, l'éducation, la santé, l'exploration des ressources naturelles, l'agriculture et la gestion des entreprises publiques. L'olm, le WUS UK, le Comité danois du wUS et le Comité international de l'organisation s'associèrent afin de composer un programme commun, le gouvernement danois apportant les fonds par le biais du wUS local, le WUS UK se chargeant des frais de déplacement via l'Administration du développement de l'outre-mer alors que le comité international du wUS accordait des

33 International wus, wus News Latin America, op. cit., p. 10 (cf. note 30 ). 
fonds provenant de la Swedish International Development Cooperation Agency (SIDCA). La sélection des candidats créa des tensions chez toutes les parties concernées, car le gouvernement du Nicaragua, outre ses demandes pour un personnel qualifié dans certains secteurs, recherchait des agents dévoués à son processus révolutionnaire. Dans le cadre de ces modalités de collaboration, le WUS UK fut partie prenante via son programme pour le retour des exilés chiliens en Amérique latine, sélectionnant les bénéficiaires sur la base de leur contribution potentielle au développement du Nicaragua ${ }^{34}$.

La promotion de ces programmes montre que, au début des années 1980, le wus avait consolidé ses positions en Amérique latine. Et de fait, à cette époque, la région bénéficiait du plus grand nombre de Comités nationaux. Alors que seulement six (soit $13 \%$ ) des 45 Comités nationaux du wus étaient basés en Amérique du Sud en $1975^{35}$, au début des années 1990, l’Amérique latine et les Caraïbes comptaient 16 Comités nationaux parmi les 39 comités restants - en d'autres termes, les Comités nationaux d'Amé-

34 Le groupe de 16 Chiliens venu du Royaume-Uni et relocalisé au Nicaragua comptait notamment le capitaine Raúl Vergara, instructeur et conseiller militaire auprès de la Force aérienne sandiniste.

35 En 1975, les comités nationaux du wus en Amérique latine étaient basés en Colombie, au Guatemala, en Haïti, au Honduras, au Nicaragua et au Pérou. Le wUs possédait aussi des contacts dans cinq autres pays, à savoir l'Argentine, la Bolivie, le Costa Rica, El Salvador et l'Équateur. II existait également 17 comités en Afrique, 9 en Europe, 17 en Asie et dans le Pacifique ainsi qu'un comité au Canada. rique latine et des Caraïbes représentaient $41 \%$ du volume total des Comités de l'organisation.

Le wUS finançait ses programmes pour une grande part en Amérique latine avec le soutien de la SIDCA et, dans une moindre mesure, avec les contributions de l'Agence finlandaise pour le développement international, le ministère des Affaires étrangères danois, le ministère des Affaires étrangères des Pays-Bas, l'UNICEF, le programme des Nations unies pour le développement (PNUD) et la fondation Ford. À mesure qu'il étendait ses objectifs et ses champs d'action, le wus était contraint de recueillir les fonds nécessaires afin de soutenir ses programmes à long terme. Alors que dans un premier temps de nombreux efforts avaient reçu l'appui financier de dons privés, les fonds versés par les agences gouvernementales dépassèrent progressivement les contributions des donateurs privés. L'affinité idéologique partagée par le wUS et par certains gouvernements (principalement des gouvernements européens en lien avec la sociale démocratie) conduisit à un soutien durable pour ses actions en Amérique latine. De façon similaire, le wus reçut également des contributions du secteur privé distribuées par des fondations philanthropiques pour certains programmes spécifiques.

De plus, la croissance du wUS - depuis la seconde moitié des années 1970 et au-delà - fut également stimulée par un facteur inattendu. Le Fonds international pour les échanges universitaires - l'International University Exchange 
Fund (IUEF) - une ONG qui fournissait une assistance et un soutien éducatif aux pays africains en voie de développement (en majeure partie l'Afrique du Sud et la Rhodésie) était jusqu'alors un concurrent du wus pour le versement des fonds, puisqu'il recevait un soutien financier d'agences de coopération internationales. À la suite d'un scandale au cours duquel il fut révélé qu'un officier militaire sud-africain avait infiltré les plus hautes sphères de l'IUEF ${ }^{36}$, les fonds des agences de coopération internationale se détournèrent de cette organisation pour affluer au WUS, lui permettant de poursuivre ses initiatives en Amérique latine.

Dans les années 1980, le wus put donc lancer d'autres programmes en faveur des réfugiés d'Amérique Centrale (Salvador, Guatemala, Honduras), qui, pour la plupart, s'installèrent au Mexique, au Costa Rica, au Panama et en Équateur. Des programmes d'aide au retour furent mis en place en direction de plusieurs pays d'Amérique du Sud dont le Chili, la Bolivie, l'Argentine et l'Uruguay. En

36 II s'agissait de Craig Williamson, qui avait pris contact avec Lars Gunnar Eriksson - alors à la tête de l'IUEF - en vue de collaborer aux programmes d'aide de l'organisation destinés aux étudiants sud-africains. II commença à travailler pour l'IUEF en 1977 et gravit les échelons jusqu'à occuper le siège de directeur-adjoint. En décembre 1979, un certain nombre d'articles publiés dans The Observer, journal londonien, révélaient que Williamson travaillait pour les services de renseignement sud-africains et avait été chargé d'infiltrer le Congrès national africain. II avait aussi pris part à des attaques, des vols et des enlèvements dirigés contre des responsables de mouvements contre l'apartheid.
1987, un programme d'aide au retour fut lancé en faveur de Haïti, suivi un an plus tard d'un plan similaire à destination du Salvador, puis en 1989, du Paraguay. Outre ses efforts traditionnels pour soutenir les réfugiés universitaires et faciliter leur retour dans leur pays d'origine, le wus mit également en place des programmes nationaux de bourses pour les groupes vulnérables - victimes de violence politique et économique - et des programmes d'émancipation des femmes, entre autres exemples. Ces projets étaient regroupés sous cinq grandes catégories: Éducation et renforcement institutionnel (pour favoriser le développement, la démocratie et les droits de l'homme); Éducation au développement et responsabilisation des femmes; Bourses et programmes en faveur des réfugiés en Amérique latine et dans les Caraïbes (aide humanitaire et formation des ressources humaines pour le développement) ; Coopération et échanges universitaires ; et enfin Droits de l'homme dans le domaine éducatif ${ }^{37}$. Les programmes du wUS indiquaient une corrélation avec les contextes politiques. Ainsi, dans des pays tels que le Pérou, le Guatemala et la Colombie, le wus déploya des programmes humanitaires, alors que ses actions au Chili étaient plutôt centrées sur des questions d'ordre politique ou éducatif.

37 International wus, Veinticinco Años de Asistencia al Desarrollo Educacional y Laboral de los Perseguidos por las Dictaduras del Cono Sur, Santiago de Chile, Coordinación Regional Para América Latina y El Caribe wUS, 1995. 
Dans les années 1990, le wUS connut une période de déclin et succomba à une sévère crise financière et administrative qui contraignit son bureau de Genève à fermer ses portes en 1995. Alors que plusieurs organismes donateurs continuèrent à soutenir ses programmes latino-américains pendant un certain temps, le désastre financier de l'organisation ne fut ni effacé, ni enrayé. Plus spécifiquement, la SIDCA, principale source de financement pour les programmes d'aide aux réfugiés latino-américains du WUS, renonça à lui verser des fonds en 1997, comme le rapportent Lennart Peck et Carlos Vilas $^{38}$.

Le wUS fut aussi, plus largement, victime du changement d'orientation des fonds versés aux fondations et aux ONG. Comme le relève Sikkink, les versements aux ONG

« accordent un grand prix à la nouveauté - ou, en termes plus critiques, ils sont assujettis à des modes. Certaines questions sont dans l'air du temps et chaque fondation digne de ce nom doit posséder son propre projet spécifique qui recevra sans aucun doute des fonds... Durant plusieurs années, les fonds versés aux organismes étaient alloués pour une grande part à l'Amérique latine et à l'Afrique du Sud. À la fin des années 1980 et au début des années 1990, quand l'Amérique latine et l'Afrique du Sud ont retrouvé le chemin de la démocratie, les versements aux organismes ont commencé à se tarir et de nouvelles ressources

38 Lennart Peck, Carlos Vilas, Servicio Universitario Mundial (WUS) en América Latina: programa de becas para refugiados, op. cit. (cf. note 16). ont été accordées en réponse à de nouvelles questions et destinées à d'autres régions du monde $»^{39}$.

Ainsi, à mesure qu'évoluait la situation politique qui avait conduit le wUS à mener des actions en Amérique latine, les nouveaux programmes qui y étaient liés - en particulier ses activités de démocratisation universitaire et ses travaux pour les droits des femmes des secteurs populaires - devaient s'autofinancer, puisqu'ils ne figuraient plus à l'ordre du jour des donateurs. Ce nouveau scénario affaiblit les opérations menées par les comités nationaux et contribua à une quasi-absence de visibilité du WUS en Amérique latine au XXI ${ }^{\mathrm{e}}$ siècle. Dans le même temps, à cause de la place de plus en plus importante accordée à l'engagement social et politique, nombre des initiatives du wUS ont été prises en charge par des agences étatiques.

\section{Réseaux, agents et habitus transnational}

Comme nous l'avons signalé au départ, ce type d'organisations opère dans toute une gamme de situations d'urgence, de contextes et de structures. Caractéristique commune et constante, il a été établi que les associations recourent au partenariat afin de répondre à des besoins spécifiques. De fait, le wUS a travaillé avec de multiples organismes pour déployer ses programmes. Si l'on s'en tient à la caractérisation

39 Kathryn Sikkink, "La dimensión transnacional de los movimientos sociales », op. cit., p. 327-328 (cf. note 22). 
formulée par Keck et Sikkink ${ }^{40}$, les spécificités organisationnelles et le maillage du wUS en font une coalition transnationale, qui présente un certain degré de coordination, de modalités formelles de contact et d'identité transnationale collective ${ }^{41}$.

Il faut opérer une distinction entre les réseaux constitués par le wuS en Europe et en Amérique latine. En Europe, cette ONG a forgé des liens avec des organismes qui apportaient leur aide aux réfugiés : partis sociaux-démocrates et de gauche, organisations à vocation sociale ou religieuse, ou encore agences internationales comme les Nations unies. En 1950, le wuS a reçu le statut de consultant du Conseil économique et social des Nations unies (ECOSOC) et a été reconnu par l'UNESCO. Il a également travaillé en lien étroit avec le Haut-commissariat aux réfugiés, l'Organisation internationale pour les migrations et la Commission pour les droits de l'homme mis en place par l'ECOSOC. Le WUS a aussi été nommé en qualité d'observateur par la Commission africaine des droits de l'homme.

En Amérique latine, en raison de l'exil forcé des intellectuels chiliens dû au coup d'État militaire de 1973, le wUS a travaillé en étroite collaboration avec le Conseil latino-américain de sciences sociales (CLACSO) à Buenos Aires en Argentine pour le programme mis en place par le wUS

40 Margaret Keck, Kathryn Sikkink, Activist Beyond Borders: Advocacy Networks in International Politics, op. cit. (cf. note 14).

41 Kathryn Sikkink, « La dimensión transnacional de los movimientos sociales », op. cit. (cf. note 22).
$\mathrm{UK}^{42}$ ainsi qu'avec des membres de la faculté latino-américaine de sciences sociales (FLACSO) au Chili, certaines organisations religieuses ou qui promouvaient les droits de l'homme (le Vicariat de la Solidarité au Chili et la Fondation de l'aide sociale des Églises chrétiennes) et la Commission évangélique latino-américaine d'éducation chrétienne (CELADEC) au Pérou. Il convient de noter que le wuS a incité ses Comités nationaux à opérer avec une certaine autonomie en élaborant leur propre réseau de contacts et en trouvant eux-mêmes leurs sources de financement. De cette manière, les Comités nationaux du wUS ont forgé des liens avec des groupes pour la défense des droits de l'homme, des universités, des organismes étudiants, des mouvements sociaux et religieux et des partis politiques. Le type de liens et de réseaux établis dépendait des caractéristiques des programmes nécessaires et mis en place. À titre d'exemple, au Chili, où les programmes ont commencé à revêtir un caractère éducatif, le contact institutionnel privilégié du wus a été l'Université académique d'humanisme chrétien, alors que, au Pérou, pays dans lequel les liens entretenus par le wUS avec d'autres universités se sont révélés plus fragiles, l'organisme travailla de concert avec l'Association pour la défense des droits de l'homme.

42 Paola Bayle, "Emergencia Académica en el Cono Sur: el programa de reubicación de cientistas (1973-1975)", Íconos. Revista de Ciencias Sociales, n 30,2008 , p. 5163. 
Les réseaux de contact du wUS prirent principalement de l'ampleur lorsque les coups d'État militaires eurent installé des dictatures dans le Cône Sud de l'Amérique latine. Les émigrés latino-américains firent connaître leur cause et eurent un impact non négligeable dans leurs pays d'accueil, procurant une aide effective dans l'élaboration de ces réseaux. Le rôle joué par les membres du wUS en voyage ou en mission en Amérique latine a été tout aussi important. Dans les deux cas, les contacts antérieurs se sont avérés déterminants et ont augmenté en nombre, car certains de ces processus politiques déclenchaient également une vague de sympathie dans les milieux progressistes européens.

Les responsables du wus ont développé des comportements et des aptitudes que l'on peut qualifier d'habitus transnational. Une fois encore, il peut être utile de distinguer au moins deux profils. D'une part, les membres européens du WUS n'ont pas rejoint cette organisation pour des raisons d'urgence personnelle ; ils y étaient plutôt poussés par d'autres motivations, comme par exemple des expériences antérieures de bénévolat auprès d'organismes universitaires ou religieux, des convictions politiques, et, dans de nombreux cas, le capital linguistique nécessaire au travail avec les réfugiés. Ils appartenaient tous à une génération de jeunes issus de la couche supérieure de la classe moyenne qui allaient au lycée ou à l'université dans les années 1960, époque à laquelle la société civile se radicalisait et où les jeunes obtenaient une plus grande visibilité et se politisaient sur fond de politiques conservatrices qui empêchaient les changements révolutionnaires en Europe même. Pour nombre de ces jeunes, l'Amérique latine représentait le lieu par excellence des utopies politiques. En travaillant pour le wUS, ces agents ont acquis les aptitudes nécessaires à la gestion et à la levée de fonds, à la menée de campagnes internationales, à l'interaction avec des agences publiques et internationales en d'autres termes, ils ont appris à exercer des pressions. Dans le même temps, ils ont témoigné d'une grande adaptabilité à la diversité géographique et culturelle en raison de leur mobilité territoriale.

Une approche ethnographique met en lumière que, après avoir travaillé pour le WUS, ces agents ont maintenu un lien avec d'autres organismes similaires - tous d'envergure internationale mettant leur habitus transnational au service d'autres causes. En raison de leurs contacts étroits avec des causes et ceux qui les défendaient, ces agents augmentaient leur capital militant, capital entendu comme un ensemble de dispositions et de compétences - un savoirfaire - subsumés dans des expériences politiques collectives et qui peut être transféré dans d'autres domaines ${ }^{43}$. Pour nombre de ces agents, il s'agissait de leur premier engagement politique.

43 Frédérique Matonti, Franck Poupeu, « Le capital militant Essai de définition ", Actes de la recherche en Sciences Sociales, $n^{\circ} 155,2004$, p. 5-12. 
D'autre part, les membres latino-américains du WUS ont aussi développé un habitus transnational, quoique de façon différente par rapport au groupe précédent et, peut-être, dans une moindre mesure. En premier lieu, ils ont rejoint le wus en raison des situations d'urgence et des besoins qui les assaillaient dans leur environnement immédiat. La plupart disposaient d'un capital militant qui, de fait, était caractéristique des intellectuels latino-américains dans les années $1970^{44}$. En second lieu, lorsque la démocratie fut restaurée en Amérique latine, ces agents poursuivirent leur carrière universitaire et politique. Un groupe a maintenu ses liens avec des universités et des organismes civiques locaux, alors qu'un autre groupe est parvenu à rejoindre le secteur public et qu'un troisième a continué à travailler avec des organisations internationales, développant davantage encore cet habitus transnational. En outre, ces agents ont acquis des compétences dans les domaines de la mise en place de réseaux, de la collecte de fonds et de la gestion de ressources financières, tout comme leurs homologues européens.

Une biographie collective détaillée permettrait une étude approfondie du rôle tenu par l'internationalisation dans la carrière professionnelle des agents associés à ce type d'organisations. En effet, il est nécessaire de déterminer si un type de capital spécifique qui s'est construit et a été accumulé dans un environnement trans-

44 Fernanda Beigel, Autonomía y Dependencia Académica: Universidad e investigación científica. Chile y Argentina (1950-1980), Buenos Aires, Biblos, 2010. national est reconnu à l'intérieur de frontières nationales. Les transferts possibles de ce capital dans d'autres domaines comme les agences étatiques et le domaine universitaire, entre autres, seraient également dignes d'intérêt. Cependant, cette étude souligne les antécédents professionnels retirés auprès d'organisations transnationales qui créent et reproduisent un type de capital spécifique, acquis par des agents qui ont été au service du wUS.

Enfin, cette étude a montré les interactions spécifiques entre des domaines nationaux et internationaux, en explorant la façon dont les opérations d'une agence internationale - comme le WUS - ont été façonnées par des justifications nationales en raison de la relative autonomie des comités nationaux. En effet, nous avons établi que chaque programme du wUS répondait aux besoins de son environnement national respectif, qui n'était exempt ni de luttes ni de différends internes. Malgré leur autonomie auto-proclamée quant à l'élaboration et à l'exécution des programmes, ce type d'organisation philanthropique dépend de fonds versés par des agences - en l'espèce, principalement par des agences publiques (agences gouvernementales ou ministères du Développement). Pour ces raisons, les secteurs nationaux qui obtiennent une ligne de crédit - pour les pays développés, cela va sans dire - imposent leurs critères et les actions sur lesquelles se concentrer, déterminant le destin de programmes et d'ong en leur accordant ou en leur refusant des fonds. Ainsi, l'arrivée du WUS en Amérique latine comme son 
Paola Bayle - Juan José Navarro

départ ont été dictés par des changements qui ont affecté les critères quant aux versements de fonds à des initiatives philanthropiques.

Traduit de l'anglais par Isabelle Vallée 